\title{
Induction and Natural Kinds Revisited
}

\section{Howard Sankey}

\section{Introduction}

In this paper, I revisit a topic about which I wrote twenty years ago. In 'Induction and Natural Kinds' I offered a metaphysical solution to the epistemological problem of induction. The solution drew on the insights of Hilary Kornblith about the relationship between induction and natural kinds. In a departure from Kornblith, I employed Brian Ellis’s account of natural kinds, which I found more plausible than the account of kinds to which Kornblith himself subscribed.

The problem of induction is a problem of circularity. Our spontaneous reaction to the question of how to justify induction is that it has been reliable in the past so will continue to be so in future. But to reason in this way is to employ induction to justify itself, which is to argue in a circle. In my own attempt to justify induction, I employ an inference to the best explanation of the reliability of induction. But inference to best explanation and induction are both forms of ampliative inference. I therefore employ ampliative inference to support ampliative inference. Hence my approach also falls foul of the problem of circularity.

In this paper, I return to the topic. I take the threat of circularity to be genuine, though perhaps it may be mitigated. I now wish to suggest that there is a way to avoid the circularity. The way to avoid the circularity is to recognize that it is the way the world is that grounds the reliability of induction, not the inference which we employ to establish the grounds of such reliability. My aim in this paper is to explain this way of avoiding the problem of induction. Before turning to that task, some background must be provided. 
In section 2 I discuss the relationship between metaphysics and epistemology as it pertains to the problem of induction. In the present context, I assume a reliabilist theory of epistemic justification, and I focus exclusively on enumerative rather than non-enumerative induction. I outline these assumptions in section 3. Section 4 presents the objection that the approach falls prey to the charge of circularity. In Section 5 I outline the relevant aspects of Kornblith's account of induction. In section 6 I briefly present Ellis's theory of kinds. In section 7, I present my own view. In section 8 I respond to the circularity objection. In section 9, I consider an objection that may be raised against my response. Section 10 presents a brief conclusion.

\section{A metaphysical starting-point}

W.V.O. Quine’s paper, 'Natural Kinds’ (1969), is an ancestor of the approach to induction that I propose. Quine argues on evolutionary grounds that our subjective similarity spacings reflect real divisions in nature. As he famously put the point, "Creatures inveterately wrong in their inductions have a pathetic but praiseworthy tendency to die before reproducing their young” (1969, p. 126). Quine recognizes that his appeal to evolutionary considerations leads in a circle. It employs the inductively based theory of evolution to provide the rationale for induction. But he declines to take the charge of circularity seriously, remarking that there is “no first philosophy” (1969, p. 127).

On one way of understanding first philosophy, epistemology is prior to metaphysics. ${ }^{1}$ We must first establish that we are able to have knowledge. Only once this is done, may we proceed to the task of determining how the world actually is. This is a traditional thought in

\footnotetext{
${ }^{1}$ One need not take epistemology to be first philosophy. In his (2009, chapter 6), Ellis argues that the ontology of scientific realism may play the role of first philosophy.
} 
philosophy familiar from Descartes's Meditations on First Philosophy. Descartes seeks to determine what may be known with certainty. He arrives at the view that certainty requires clear and distinct ideas. Only then does he turn to the question of how to show that we have knowledge of the external world. Thus, for Descartes, epistemology is first philosophy. He seeks to show that we are able to have knowledge before turning to the nature and existence of the external world.

I propose an inversion of this Cartesian order. In at least one instance, metaphysics is prior to epistemology. We must first establish that something metaphysical is the case. Based on this, we may proceed to solve the epistemological problem.

I wish to argue that we should adopt a metaphysical stance as starting-point before turning to the problem of induction. ${ }^{2}$ The problem of induction is how to provide a noncircular justification of induction: how may we justify induction without relying upon induction itself in the course of the justification? My suggestion is that it is the way the world is that makes induction reliable. The world contains natural kinds whose members have essential properties. Possession of essential properties by the members of natural kinds is what makes inductive inference reliable. The trick is to show that this appeal to the way the world is does not proceed in a circular manner.

\section{Reliabilism and enumerative induction}

I will assume a reliabilist account of epistemic justification. In seeking to justify induction, I aim only to provide an account of the reliability of induction. For present purposes, if a satisfactory account of the reliability of induction can be given, that suffices to justify

\footnotetext{
2 Michael Devitt's slogan "put metaphysics first" is apposite here.
} 
induction. There is no need to go on to argue that we are justified to employ a reliable form of inference or belief-formation. ${ }^{3}$

To simplify matters, I focus on enumerative induction. For example, suppose we infer that all platypuses have webbed feet because all of the platypuses that we have observed have had webbed feet. This is an instance of enumerative induction. In such an inference, we generalize from a limited number of observed items to a claim about all members of the kind to which the items belong. My thesis is that such enumerative induction is grounded in natural kinds. It is because all members of the natural kind platypus have webbed feet that the inductive inference from observed to unobserved platypuses yields a correct conclusion. ${ }^{4}$

For present purposes, the question of the applicability of my account to more sophisticated forms of induction may be safely set to one side. This should not be taken to suggest that all induction is enumerative. Nor is it to suggest that non-enumerative forms of induction are unimportant. I seek to provide a justification for induction, not an account of all of our inductive practices. If the approach succeeds in the case of enumerative induction, it may plausibly be extended to other forms of induction. If the approach fails to do justice to enumerative induction, it is unlikely to provide suitable warrant for non-enumerative forms of induction.

This is simply a matter of taking things one step at a time. If enumerative induction may be justified in the way that I propose, then it is worth exploring the implications of the

\footnotetext{
3 Those of a more internalist persuasion may baulk at my use of the term 'justification' in the context of a generally reliabilist approach to induction. To partially assuage such qualms, I will simply mention Ernest Sosa's suggestion that there is a further sense of 'justify' in addition to the idea of justification of a belief on the basis of a state with propositional content (Sosa 1980, p. 7). An action may be justified by means of its consequences rather than on the basis of some propositional content. It is this latter sense of 'justified' that is apposite here.

4 It may be objected that having webbed feet is not an essential property of duckbill platypuses. If a platypus were born without webbed feet, it would still be a platypus. This may be granted without compromising the basic point. Rather than webbed feet, we may substitute instead a more suitable candidate for essential property of platypus, perhaps a particular genetic code. The induction about webbed feet would go through because having webbed feet is a property that depends upon the essential property.
} 
approach for less basic forms of induction. If the approach fails in the case of enumerative induction, there is little hope of extending it to other forms of induction.

\section{The charge of circularity}

'Induction and Natural Kinds' originally appeared in 1997. At the time, I was concerned that my approach might fall prey to the charge of circularity. Hence I explicitly addressed the issue in the paper. A revised version of the paper was included in my book, Scientific Realism and the Rationality of Science (2008). In the revised version, I sought to improve the approach by drawing on work by David Papineau. Though I remain sympathetic to Papineau's view, I now have a further proposal to make, which has been prompted by some critical comments by Stathis Psillos.

In his review of Scientific Realism and the Rationality of Science, Psillos presents a number of objections to my approach to induction. The chief objection is that the approach employs ampliative inference to justify ampliative inference, and thereby fails to avoid circularity. Psillos writes as follows:

Sankey's gambit is smart: it assumes the existence of natural kinds to explain the reliability of induction. Should it work, it promises to avoid the well-known charge of circularity. The move from the success of scientific methodology to the existence of natural kinds is abductive: the existence of natural kinds is taken to be the best explanation of the success of science. If so, the existence of natural kinds can do apparently non-circular work in justifying induction.... it is wrong to believe that IBE can bypass the problem of induction - since the problem concerns, at bottom, the very idea of an ampliative but rational method. IBE has no atemporal warrant - as Hume in effect observed when he criticized a standard appeal to active powers to justify induction.... Nor is it obvious that essential properties explain the reliability of induction in a non-circular way, since, as Sankey himself notes, 'good inductive inferences project essential properties, whereas bad ones project accidental properties'. Unless there is an independent way to classify inductions into good and bad ones, essentialism cannot ground the reliability of induction. (Psillos 2009, p. 682) 
In this passage, Psillos raises a number of critical points against my position. I will focus on what I understand to be the central objection. I take the central objection to be that my approach fails to avoid the charge of circularity.

In the revised version of 'Induction and Natural Kinds', I sought to forestall the circularity objection by adopting an approach with which Psillos appears to be sympathetic (cf. Psillos 1999, p. 82). This is the approach adopted by David Papineau in his inductive defence of the reliability of induction (1992). Papineau argues by induction from the past reliability of induction to its general reliability. Against the charge of circularity, Papineau appeals to the distinction between premise and rule circularity. A premise circular argument contains the conclusion itself in the premises. A rule circular argument uses a rule of inference (e.g. induction, deduction) to support the rule itself. The conclusion that induction is reliable does not appear as a premise in the argument for the reliability of induction. Papineau's inductivist defence of induction is not therefore premise circular. But it is rule circular.

I continue to regard the idea of rule circularity as an important tool to be employed in the inductive justification of induction. But I now wish to propose another way to avoid the charge of circularity. My goal here is to explain this other way. Before I turn to that, let me describe the approach to induction in greater detail. The approach is not new. Nor is it mine alone.

\section{Hilary Kornblith on induction}

My approach to the problem of induction is related to the venerable idea that our use of induction rests upon a principle of the uniformity of nature. In my view, induction is reliable 
because the world makes it so. In effect, I endorse a version of the principle of uniformity according to which induction is justified as a result of nature being uniform. This principle is not something that we know a priori to be true. Nor is it to be thought of as a logical presupposition of inductive inference. The principle is an empirical claim about the nature of reality. It is an a posteriori metaphysical claim.

I draw explicitly on the position developed by Hilary Kornblith in his book, Inductive Inference and its Natural Ground (1993). Kornblith presents an account of the reliability of inductive inference which rests on two key claims. On the one hand, the world has a natural kind structure. That is, the world contains individual items which are members of natural kinds. On the other hand, our minds reflect the natural kind structure of the world. We conceptualize the world in a way that is sensitive to its natural kind structure. Moreover, our inductive inferences presuppose the existence of such a structure. Given the way our minds reflect the kind structure of the world, there is a "dovetail fit" between mind and the world. This dovetail fit explains the reliability of inductive inference.

In my account of induction, I emphasize the role of natural kinds in explaining the reliability of induction. I focus primarily on Kornblith's view that natural kinds provide the ground for induction rather than on his account of how the human mind fits with the natural kind structure of the world. But Kornblith is surely right that an account must be given of how we conceptually grasp natural kinds and reason inductively in a manner that is informed by the natural kind structure of the world. I do not have anything substantive to say about this aspect of the position. I shall simply assume that an approach along the lines developed by Kornblith is well-motivated and in broad terms correct.

What sort of argument does Kornblith provide for the approach? Kornblith argues that the natural kind structure of the world is the best explanation of the reliability of 
induction. His use of this form of argument is especially apparent when he considers the role played by natural kinds in inductive inference in the sciences. In that context, his argument takes a form that is familiar from the literature on scientific realism:

If the scientific categories of mature sciences did not correspond, at least approximately to real kinds in nature, but instead merely grouped objects together on the basis of salient observable properties which somehow answer to our interests, it would be utterly miraculous that inductions using these scientific categories tend to issue in accurate predictions. Inductive inferences can only work, short of divine intervention, if there is something in nature binding together the properties which we use to identify kinds. Our inductive inferences in science have worked remarkably well, and, moreover, we have succeeded in identifying the ways in which the observable properties which draw kinds to our attention are bound together in nature. In light of these successes, we can hardly go on to doubt the existence of the very kinds which serve to explain how such successes were even possible. (1993, pp. 41-2)

In this passage, Kornblith's inference to the best explanation of reliable inductive inference in science bears a clear and striking resemblance to the "no miracles" argument for scientific realism made famous by Hilary Putnam. Given this reliance on an ampliative form of inference, however, Kornblith’s approach would appear to be vulnerable to the same charge of circularity as is my own version of the approach. ${ }^{5}$

As indicated, Kornblith's account of induction rests on the co-occurrence of properties among members of a natural kind. In order to explain such co-occurrence of properties, Kornblith adopts the homeostatic property cluster (HPC) account of natural kinds proposed by Richard Boyd. On Boyd's account, natural kinds possess groups of properties which enter into a relation of homeostatic equilibrium:

A natural kind is a cluster of properties which, when realized together in the same substance, work to maintain and reinforce each other, even in the face of changes in the environment. (1993, p. 35)

\footnotetext{
5 As Kornblith does not address the charge of circularity, it is difficult to determine how he might respond to it. It is entirely possible that his attitude toward the charge might parallel Quine's naturalistic dismissal of the issue.
} 
On the HPC account, only certain groups of properties are able to co-occur in stable assemblages. It is this fact about the way in which properties may be arranged in only a limited number of ways that provides the basis for reliable inductive inference:

Because there are natural kinds, and thus clusters of properties which reside in homeostatic relationships, we may reliably infer the presence of some of these properties from the presence of others. In short, natural kinds make reliable inductive inference possible, because were it not for the existence of these homeostatic clusters, the presence of any set of properties would be fully compatible with the presence of any other. (1993, p. 36)

For Kornblith, the way in which certain groups of properties are able to reside together in homeostatic equilibrium is what provides the metaphysical underpinning of inductive inference. This is why he thinks of natural kinds as providing "the natural ground of inductive inference” (1993, p. 36).

No doubt, the HPC account may be fruitfully applied to natural kinds within the life sciences. It is less clear that it may be extended to non-biological kinds. The account rests on a metaphor that applies in the case of living organisms. It does not readily generalize to physical entities other than organisms. For this reason, I choose not to follow Kornblith in adopting Boyd's account of natural kinds.

\section{Brian Ellis on natural kinds}

My own preference is to adopt an essentialist theory of natural kinds of the kind proposed by Brian Ellis. Such an account seems to me to provide a more solid foundation for inductive inference than does the HPC account. ${ }^{6}$

\footnotetext{
6 I do not wish to rule out the possibility that a position like the HPC account might be combined with the essentialist view of Ellis's that we are about to consider. The HPC account seems to be designed for biological rather than physical kinds. By contrast, Ellis's account is designed primarily for physical and chemical kinds. Ellis considers biological kinds to be clusters of microspecies (2002, p. 31), which suggests there might be room for reconciliation with the Boydian view.
} 
According to Ellis, individual entities such as electrons belong to natural kinds. But individual entities are not the only things that belong to natural kinds. In addition to natural kinds of entities or objects, there are also properties such as charge and mass, as well as relations such as gravitational attraction or chemical bonding. Moreover, there are natural kinds of events and processes, such as chemical reactions or radioactive decay.

The world in which we live is highly structured. This is largely due to the fact that it is divided up into categorically distinct natural kinds. Natural kinds have objectively existing boundaries which we discover. They do not overlap or fade into each other. The borderline between them is not an arbitrary or blurred one that depends on a human classificatory decision or convention. The only way that kinds may overlap is by way of a relation of inclusion such as the relation of genus to species. The system of natural kinds forms a hierarchical structure. More specific kinds belong to more general kinds. General kinds possess essential properties which are also found in more specific kinds. The specific kinds within a general kind all possess the essential properties of the general kind.

For Ellis, the essential properties of natural kinds are intrinsic properties shared by all members of the same kind. The essential properties of natural kinds of things are dispositional. They have "the nature of powers, capacities and propensities" (1999, p. 19). Natural kinds are characterized by the intrinsic causal powers of the things that belong to those kinds. This permits a connection to be drawn between laws of nature and natural kinds. Laws of nature describe the essences of natural kinds. They are statements that are made true by the causal powers of things that belong to natural kinds. The truth-makers for laws of nature are the causal powers or intrinsic dispositions of natural kinds of things. Because the causal powers or dispositions are essential properties of natural kinds, the laws of nature are not contingent. They are metaphysically necessary, grounded in the essential properties of natural kinds of things. 


\section{My approach}

On the view that I propose, induction is justified because nature is uniform. There is a sense, as we have seen, in which my approach involves a principle of the uniformity of nature. But the principle of uniformity is not to be understood in the way such principles are usually understood. It is not an overarching or blanket statement to the effect that the future resembles the past. Nor is it simply a global statement that nature is uniform.

The principle relates specifically to the essential properties of natural kinds. On this version of the principle, nature is uniform in the precise sense that there are natural kinds whose members all possess a shared set of essential properties. In light of the relation that obtains between natural kinds and laws of nature, to say that nature is uniform is to say that it is governed by laws of nature. On the essentialist view that I take over from Ellis, laws of nature are grounded in the causal powers that are the essential properties of natural kinds of things.

What I wish to suggest is as follows: when we use induction to form a belief about the future we are justified in doing so because nature is in fact uniform. It is uniform in the sense that there are natural kinds of things which possess sets of essential properties. All members of a kind have the same essential properties. Unobserved members of a kind possess the same essential properties as observed members of the kind. The fact that observed and unobserved members of a kind possess the same essential properties is what makes induction reliable. When we predict that an unobserved object will have an essential property that observed objects of the same kind have our prediction will be correct. It will be correct because all members of the kind have essential properties in common. It is because all members of the kind have the property in common that our prediction is correct. 
Of course, the conclusion of an inductive argument need not be correct. On the present account, one way in which an inductive inference may lead to a false conclusion is for essential properties to be mistakenly identified as such. If a non-essential property were mistakenly taken to be an essential property, future unobserved members of the kind may fail to have the property mistakenly thought to be essential to the kind. In such a situation, the prediction that a future unobserved member of the kind will possess the property in question may be mistaken.

As so far stated, the account requires two qualifications. The first relates to the apparent restriction of inductive inference to essential properties of natural kinds. The second relates to the question of how inductive inference may be employed with respect to items that do not belong to natural kinds.

With regard to the first point, I have presented the account in terms of the essential properties of natural kinds. But this is an oversimplification. Inductive inference need not be restricted to essential properties. There may be non-essential properties that depend on essential properties in a systematic way. Where there are non-essential properties that depend in a systematic way on essential properties, it may be possible for there to be reliable inductive inference that ranges over such dependent non-essential properties.

To take a particular example, being black is unlikely to be an essential property of ravens. In the case of ravens, the property of being black depends in some systematic way on facts about the genetic make-up of ravens that typically give rise to black pigmentation. The essential properties of ravens will likely be found within the genetic code of ravens. ${ }^{7}$ Hence, the reliability of an inductive inference about the blackness of ravens is not due to the fact

\footnotetext{
${ }^{7}$ A qualification may be in order. Arguably, being a bird is essential to being a raven. Having a beak, feathers and wings is essential to being a bird. This may or may not reside in some bit of genetic code common to all birds.
} 
that blackness is an essential property of ravens. It is due to the fact that the blackness of ravens depends in some systematic way on features of the genetic code of ravens that constitute the essential properties of ravens.

Second, as so far presented, my account of induction applies to inductive inference about members of natural kinds. But we routinely infer by induction about members of nonnatural kinds, such as trains, trams and airplanes. Surely, inductive inference is not restricted to the essential properties of natural kinds and the non-essential properties that systematically depend upon the essential properties of natural kinds. We are perfectly able to reason by induction about items that do not belong to natural kinds. ${ }^{8}$

In the original development of the approach, I was inclined to adopt an uncompromising stance with respect to this issue. I initially took the view that induction about entities that do not belong to a natural kind may only be reliable to the extent that the entities are composed of parts that do belong to natural kinds. It is only in virtue of the fact that the component parts consist of material belonging to natural kinds that inference about members of non-natural kinds may be reliable. I found encouragement for this stance in a remark due to T.E. Wilkerson: "because there are no very specific real essences that make rubbish rubbish, and tables tables, I cannot even in principle make sound inductive projections about rubbish as such or tables as such” (1995, p. 32). Wilkerson's suggestion was that only natural kind predicates are projectible and thus able to be employed in inductive inference. ${ }^{9}$ This seemed, in turn, to suggest that reliable induction might only be

\footnotetext{
${ }^{8}$ The objection may be put another way. Induction is a non-deductive form of inference in which the premises provide support for the conclusion but do not deductively entail the truth of the conclusion. Nothing about the definition of induction implies that induction must be restricted to natural kinds.

${ }^{9}$ Wilkerson's mention of projectibility brings out another attractive feature of the present approach, namely, that it promises to resolve Goodman's problem of the grue emeralds. But it may not all be plain sailing. As Bruce Langtry has pointed out to me, 'grue' is defined in terms of 'green' and 'blue' which may be natural kind predicates. So it is not immediately clear how appeal to natural kind terms does solve the grue problem.
} 
grounded in natural kinds, since it is only the regularity built into natural kinds that may provide the basis for such reliability.

The problem with such an uncompromising stance is that there are cases of apparently sound inductive inference not underpinned by the existence of a natural kind. Apart from cases in which we infer inductively about items that belong to non-natural kinds, we may also make legitimate inductive inferences about singular items considered simply as individual things rather than as instances of a kind. ${ }^{10}$ Because there are cases of reliable induction for which the existence of natural kinds does not appear to be responsible, a less uncompromising stance may be appropriate. Hence, I wish only to assert that my account of induction is able to explain the reliability of an important and presumably large class of cases of inductive inference, namely, those in which induction applies to members of natural kinds, as well as to items consisting of natural kinds which are responsible for the regularity picked out by the induction. It remains to be explained how and why induction is reliable in cases where the presence of natural kinds plays no apparent role.

\section{The objection again}

I now return to the problem that this paper seeks to address. As we have seen, my aim is to provide an account of the reliability of inductive inference and thereby to justify induction. Does this approach avoid the problem of induction? According to Psillos, my approach employs an ampliative inference to argue in support of an ampliative inference. If this is right, the approach seems not to avoid the charge of circularity at all.

10 For example, we may infer from the fact that a particular potted plant has thrived after being watered that it always thrives after being watered. Such an inductive inference need make no reference to the kind to which the plant belongs, and, indeed may be restricted specifically to the one plant under consideration. (I owe this example to Greg Restall.) 
Let me state the objection more precisely. The argument for the existence of natural kinds proceeds by inference to best explanation (IBE). A similar argument is employed to support the claim that induction is reliable because it is grounded in natural kinds. But IBE and induction are both forms of ampliative inference. So an ampliative inference is employed to justify an ampliative inference. The circularity is immediately apparent.

As mentioned previously, I earlier sought to forestall this objection by appeal to Papineau's distinction between rule and premise circularity. Though I continue to hold that this distinction has a role to play in a reliabilist justification of induction, I now favour another response to the circularity objection. Put simply, the response is that the IBE is not what does the justificatory work in the justification of induction. It is the natural kinds that provide the justification for induction.

To develop the point in more detail, it is important to reflect upon the role played by IBE in the account. There are two places where IBE is employed. First, an IBE is employed to argue for the existence of natural kinds. Second, an IBE is used to argue that the existence of real kinds in nature is what makes induction reliable. But notice that on this account the reliability of induction does not itself depend upon the IBE. What underpins the reliability of induction is not the IBE. It is the natural kind structure of the world that makes induction reliable. IBE has nothing to do with it.

To see this, suppose that we correctly employ induction to predict that a previously unobserved member of a kind will in future be found to have some specific property. On my account, the explanation of why the induction leads to a correct prediction is that it correctly picks out a real pattern in nature. Thus, it is the way the world is that makes the inference reliable. It is not the inference to the best explanation of the reliability of induction that 
makes the induction itself reliable. The reliability of the inductive inference does not depend upon the IBE.

The point may be presented in another way. It may be illustrated in terms of a once popular distinction in the philosophy of science. Karl Popper and Hans Reichenbach drew a distinction between the context of discovery and the context of justification. They distinguished between the way in which one arrives at a theory (the context of discovery) and the way in which the theory is justified (the context of justification). The initial inspiration or creative insight that gives rise to a theory need have nothing to do with the tests or experiments which provide empirical evidence on the basis of which the theory is to be accepted.

A similar point applies to the present approach to induction. IBE is employed to argue that the world has a natural kind structure, and that this structure underlies the reliability of inductive inference. This is the context of discovery. We employ IBE in the context of discovery to argue for an account of the natural ground of induction. But our use of IBE to argue for the account is not what provides the ground for induction. What justifies our use of induction is the fact that the world is a certain way. It is the natural kind structure of the world that makes induction reliable. In short, IBE is how we discover the ground of induction. It is not what grounds the induction. ${ }^{11}$

In sum, I propose an argument to the effect that induction is reliable, and so justified, because the world has a natural kind structure that makes induction reliable. If I am right, the approach is not circular. It is not circular because the IBE is not what grounds induction. It

\footnotetext{
${ }^{11}$ I do not think that the analogy between the two context distinction and the present approach to induction is a perfect analogy. But the distinction between the two contexts helps to illustrate the distinction between, on the one hand, the argument for the account of reliable induction and, on the other hand, the natural kind structure that underlies the reliability of induction and thereby provides the warrant for induction.
} 
is the world that grounds induction. The only role played by IBE is to discover that natural kinds are what make induction reliable.

\section{A further objection}

In the previous section, I attempted to meet the charge of circularity by arguing that the justification of induction rests on the natural kind structure of the world rather than upon the IBE given on behalf of this account of induction. I will now consider an objection that might be raised against this response to the charge of circularity. The objection might be presented in the following terms:

“Granted, if natural kinds exist, induction will be reliable. But we must use IBE to arrive at a justified belief in the existence of natural kinds. Moreover, we must use IBE to justify the belief that the reliability of induction is based on natural kinds. This means that the justification of induction depends upon an IBE about the reliability of induction. So, in the end, you use ampliative inference to justify induction.”

On the view that I have proposed in this paper, induction is justified because the existence of natural kinds makes inductive inference reliable. As I have pointed out, an IBE is employed to argue for this view of the warrant of induction. But I wish to deny that the IBE employed in the argument plays the role in justifying induction that the objection assumes it to play.

The objection is based on an internalist assumption that we must form a justified belief in the reliability of induction in order to be justified in our use of induction. ${ }^{12}$ But I do

12 I interpret the notion of justification employed in the objection in internalist terms. However, the notion of justification could be interpreted in reliabilist terms. If so, it is not clear that the objection poses a serious threat 
not see why we must form such a belief in order to be justified in the use of induction. The existence of natural kinds would render our use of induction reliable even if we did not perform the IBE and thereby come to hold the present view of induction. In order for use of induction to be justified, there is no need for us to know or to justifiably believe that induction is warranted by the existence of natural kinds.

To employ the above internalist assumption against the position that I have presented begs the question against the reliabilist stance adopted here. But, quite apart from that, the objection relies upon a contentious view about what is required for justification. The objection relies on an assumption similar in spirit to the infamous KK-thesis that in order to know we must know that we know. In particular, it assumes that in order be justified in our use of induction we must hold a justified belief that induction is justified. This in turn assumes that in order to be justified in a belief we must justifiably believe that we are justified in the belief.

It would be wrong to reject such an assumption out of hand. At the same time, it would be wrong to adopt the assumption without strong positive arguments in its favour. More generally, the assumption is so fundamentally at odds with the approach adopted here that it is more than just an objection. It is a wholesale rejection of the approach to the problem of induction that I have sought to sketch in this paper.

\section{Conclusion}

In this paper, I have attempted to respond to the charge of circularity raised by Stathis Psillos against my reliabilist justification of induction. Against the charge that my account employed

to the approach. Nor, however, is it clear that the assumption on which the objection is based need be granted even if justification is understood in reliabilist terms. 
ampliative inference to justify an ampliative inference, I have argued that what grounds induction is not the IBE presented for the reliabilist account of induction. It is the natural kind structure of the world that makes induction reliable. Given this, the circularity may be avoided.

I conclude in programmatic terms. As indicated, I have presented the position as an account of the reliability of enumerative induction. If the account can be sustained, then it may be worthwhile exploring the prospects of extending the approach to non-enumerative forms of induction. We have also seen that there appear to be cases of inductive inference which do not apply to natural kinds. It remains to be seen whether the account may be extended to cases where induction seems not to be applied to natural kinds or whether some alternative approach must be developed for cases in which induction does not apply to natural kinds. These are topics for future work which must be addressed if the present approach is to be developed in greater detail.

References

Ellis, Brian (1999), ‘Causal Powers and Laws of Nature’, in H. Sankey (ed.), Causation and Laws of Nature, Kluwer Academic Publishers, Dordrecht, pp. 19-34

Ellis, Brian (2001), Scientific Essentialism, Cambridge University Press, Cambridge

Ellis, Brian (2002), The Philosophy of Nature, Acumen Press, Chesham

Ellis, Brian (2009), The Metaphysics of Scientific Realism, Acumen Press, Durham 
Kornblith, Hillary (1993), Inductive Inference and its Natural Ground, MIT Press, Cambridge, Mass.

Papineau, David (1992), 'Reliabilism, Induction and Scepticism’, Philosophical Quarterly 42, pp. $1-20$

Psillos, Stathis (1999), Scientific Realism: How Science Tracks Truth, Routledge, London and New York

Psillos, Stathis (2009), Review of Scientific Realism and the Rationality of Science, Australasian Journal of Philosophy, 87: 4, pp. 681-684

Quine, W.V.O. (1969), 'Natural Kinds', in Ontological Relativity and Other Essays, Columbia University Press, New York, pp. 114-138

Sankey, Howard (1997), 'Induction and Natural Kinds’, Principia 1: 2, pp. 239-254

Sankey, Howard (2008), Scientific Realism and the Rationality of Science, Ashgate, Aldershot

Sosa, Ernest (1980), 'The Raft and the Pyramid: Coherence versus Foundations in the Theory of Knowledge’, Midwest Studies in Philosophy, 1980 (5: 1), pp. 3-26

Wilkerson, T.E. (1995), Natural Kinds, Avebury, Aldershot 